\title{
Experimental and Numerical Investigation of Fluid Flow in Hydraulic Filters
}

\author{
Y. S. Korkmaz ${ }^{1}$, A. Kibar ${ }^{2 \dagger}$ and K. S. Yigit ${ }^{3}$ \\ ${ }^{1}$ Altan Hydraulic Engineering Industry and Trade Corporation, Istanbul, 34956, Turkey \\ ${ }^{2}$ Department of Mechanical and Material Technologies, Kocaeli University, Uzunciftlik Nuh Cimento \\ Campus, 41180, Kocaeli, Turkey \\ ${ }^{3}$ Department of Mechanical Engineering, Kocaeli University, Umuttepe Campus, 41380, Kocaeli, Turkey
}

†Corresponding Author Email: alikibar@kocaeli.edu.tr

(Received April 14, 2021; accepted September 17, 2021)

\begin{abstract}
Hydraulic systems are extensively used in industries. However, these systems must be free of contaminants to ensure their durability. When the contaminants entering the system are not removed with a suitable filter, sensitive parts such as pumps, motors, and actuators would be damaged. Therefore, hydraulic filters are critical elements in hydraulic systems. In this study, the flow and pressure drop in hydraulic filters were investigated experimentally and numerically. Although the main function of this device is to filter oil, it has many other functions in the system. Experiments were performed at eight Reynolds numbers in the range of $1250-2350$ at a constant viscosity. In the experiments, the pressure between the inlet and outlet of the filter was measured differently. The numerical results were used for detailed analysis of the flow after experimental verification. The analyses were performed using eight Reynolds numbers at laminar boundaries to examine the flow in the hydraulic filter. The results show that all surface areas of the filter element are not used homogeneously for fluid passage. The resultant pressure drop is due to the Dean vortex formed at the outlet of the hydraulic filter. The findings of this study can help better understand the flow recirculation regions that produce pressure drops and contaminant accumulation regions throughout a hydraulic filter from the inlet to the outlet of the flow path.
\end{abstract}

Keywords: Hydraulic filter; Pressure drop; Numerical study; Porous media; Darcy-Forchheimer's law.

\section{NOMENCLATURE}

\begin{tabular}{|c|c|c|c|}
\hline $\begin{array}{l}e \\
f b\end{array}$ & filtration efficiency & $P_{v}$ & $\begin{array}{l}\text { porous viscous resistance } \\
\text { coefficient }\end{array}$ \\
\hline$I$ & unit tensor & $v$ & velocity of the fluid \\
\hline$k$ & Darcy permeability & & \\
\hline$L$ & length of the filter & Greek symbols & \\
\hline$N_{d} D$ & $\begin{array}{l}\text { numbers of particles in the } \\
\text { downstream area }\end{array}$ & $\beta$ & filtration beta ratio \\
\hline$N_{d} U$ & $\begin{array}{l}\text { numbers of particles in the } \\
\text { upstream area }\end{array}$ & $\mu$ & $\begin{array}{l}\text { dynamic viscosity } \\
\text { inertial factors }\end{array}$ \\
\hline$\Delta P$ & pressure differences between & $\rho$ & fluid density \\
\hline$P_{i}$ & $\begin{array}{l}\text { the upstream and downstream } \\
\text { porous inertial coefficient }\end{array}$ & $\tau$ & viscous strain tensor \\
\hline
\end{tabular}

\section{INTRODUCTION}

As with all systems, energy efficiency in hydraulic systems is one of the most important issues and needs to be considered. Many factors cause energy loss and reduce the efficiency of hydraulic systems. Every component used in the system

causes energy loss. Therefore, these components should be carefully designed. Among these components, hydraulic filters are one of the most important ones for hydraulic systems.

A critical element in hydraulic systems is the hydraulic oil, the main function of which is the transmission of fluid energy. The hydraulic oil also 
has additional functions, such as lubrication of moving parts, heat transfer, sealing of moving parts, corrosion protection, and contaminant discharge (Olszak et al. 2020). The failure to perform one of these functions may result in components corrosions, orifice clogs, deterioration of the chemical properties and viscosity of the oil, and loss of oil additive properties. Therefore, at least one filter is needed in all of hydraulic systems to protect the systems and their components. The failure of hydraulic systems due to solid particulate contaminants accounts for $70 \%-80 \%$ of all failures caused by oil contamination (Zhang et al. 2018).

Contaminants can enter hydraulic systems in different ways, such as through actuator seals, from wear on the system components, during maintenance, and through the addition of fresh oil to the system without filtering during an oil change. When these contaminants are not removed from the system, the efficiency of the hydraulic system decreases and may cause major malfunctions. Hydraulic filters may vary in their sensitivity and resultant pressure loss depending on the system location and function. The power of hydraulic pumps is dependent not only on the required force but also on the losses, specifically due to the pressure drop in the system. Therefore, the pressure losses of components such as filters should be as low as possible.

The hydraulic filter traps and collects particles from the oil and keeps the oil clean to protect the system elements from abrasion and prevent machine errors. The performance of each hydraulic filter is determined by its particle retention capacity. The specification of the pressure drop, which is mainly due to the filter element, is critical in determining the contamination rate of the filter. A primary feature of filtering media is their resistance to fluid flow, which is directly related to the physical structure of the filter elements (Momin et al. 2017).

Hydraulic filters can be categorized into four types, namely those used in the pressure, suction, return, and off-lines. A filter element used in the pressure line has a more precise permeability, whereas the sensitivity of the filters used in the suction line is low, allowing large particles to pass through. Offline filters can be installed and maintained without the need to shut down the system. Moreover, these filters are mostly preferred in the hydraulic systems because of their ability to filter for 24 hours even when the system is not working. Fig. 1 shows the oil flow in a hydraulic filter. While the oil entering the filter housing passes through the filter element, the contaminants in the oil are trapped by the filter. Then, the oil is discharged from the filter to the system.

For efficient hydraulic filtering, the following conditions are needed:

$>$ Maximum filtering area

$>$ Minimum pressure drop

$>$ Uniform flow

$>$ Minimal contamination-clogging rate
$>$ Optimized filtration medium size

$>$ No secondary flows and recirculation

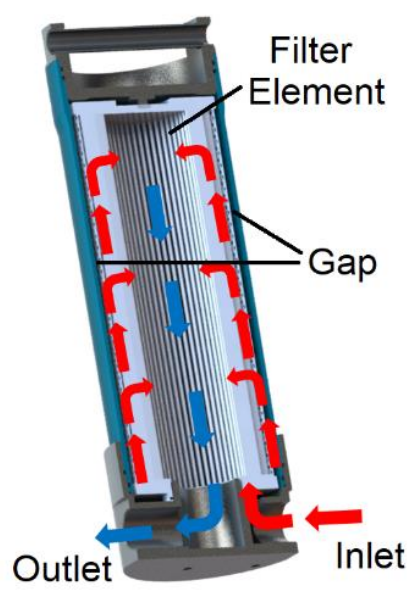

Fig. 1. Fluid flow in a hydraulic filter.

The primary factor in the selection of filter elements is the filtration beta ratio $(\beta)$, which indicates the efficiency of the filter element. $\beta$ is determined by tests performed according to the ISO 16889 standard and is defined as the ratio of the number of upstream area particles to the number of downstream area particles, as defined in Eq. (1) (Alan and Tercan 2013; Wakeman 2007).

$$
\beta=\frac{N_{d} U}{N_{d} D}
$$

where $N_{d} U$ and $N_{d} D$ are the numbers of particles in the upstream and downstream areas, respectively.

The relationship between $\beta$ and the filtration efficiency $(e)$ is expressed in Eq. (2).

$$
\beta=\frac{1}{1-\left(\frac{e}{100}\right)}
$$

As indicated in Eq. (2), the higher the filtration rate, the higher the $\beta$ value. The relationship between $\beta$ and $e$ is presented in Table 1 (Alan and Tercan 2013). Using a filter element with a higher beta ratio in hydraulic systems will reduce pressure losses, thereby increasing efficiency.

Many studies have investigated the flow in hydraulic components such as elbows (Korkmaz et al. 2021; Perumal and Ganesan 2016; Zahedi et al. 2019), pipes (Düz 2019), bends (Reghunathan Valsala et al. 2019; Rinaldi et al. 2019; Tarodiya et al. 2020), Tjunctions Ŝtigler et al. 2012) and valves (Okhotnikov et al. 2020). Besides, studies on hydraulic filters have mainly examined the design (Momin et al. 2017), optimization (Tekelioğlu et al. 2020), clogging properties (Eker et al. 2016), and strength (Momin et al. 2017), in addition to fatigue (Park 2002), and contamination (Singh et al. 2012; Zhang et al. 2018). However, to the best of our knowledge, no numerical study has been conducted to analyze the flow through a hydraulic filter. 
Table 1. Relationship between the beta ratio and filtration efficiency (Sutherland 2008).

\begin{tabular}{|c|c|}
\hline Beta ratio $(\boldsymbol{\beta})$ & Efficiency $(\boldsymbol{\%})$ \\
\hline 1 & 0 \\
\hline 2 & 50 \\
\hline 10 & 90 \\
\hline 20 & 95 \\
\hline 50 & 98 \\
\hline 100 & 99 \\
\hline 1000 & 99.9 \\
\hline 10000 & 99.99 \\
\hline
\end{tabular}

Majdan et al. (2017) proposed three different filtering systems using a tractor hydraulic circuit and confirmed their designs via experimental studies. They stated that the reduction in the pollution level increased the capability of filtration systems designed for agricultural tractors. Park (2002) specified that the selection of appropriate material is vital for ensuring the fatigue strength of hydraulic filters. Gorle et al. (2018) experimentally investigated the effect of the oil temperature, flow rate, and contamination level on the performance of a hydraulic filter. They stated that filtration rates increase as the viscosity decreases with increasing temperature; therefore, more time is required to obtain the pressure upstream of the filter element.

Darcy's equation is commonly used to determine the pressure drop through a porous medium. The filter element has a pleated design to allow for an increased surface area through which the fluid passes.

According to the Darcy model in Eq. (3), the relationship between the velocity/viscosity and pressure drop through a porous medium is linear. The validity of Darcy's law is limited by steady-state, laminar, viscous $(\operatorname{Re}<1)$, and incompressible Newtonian flow, neglecting the inertial effect (Lasseux and Valdés-Parada 2017).

$$
\frac{\Delta P}{L}=\left(\frac{\mu}{k}\right) v
$$

where $\Delta P$ is the pressure difference between the upstream and downstream pressures, $k$ is the Darcy permeability. $v$ is the velocity of the fluid. $\mu$ is the dynamic viscosity of the liquid, and $L$ is the length of the filter.

Inertial forces become significant as the velocity of the flow (i.e., Reynolds number) increases. Equation (4), known as the Forchheimer equation, can be used to define the pressure drop through a porous medium for high flow velocities. Therefore, the steady, laminar, and nonlinear (i.e., $\Delta P$ and $v$ ) flow may be defined using the Forchheimer equation.

$$
\frac{\Delta P}{L}=\varphi \rho v^{2}+\left(\frac{\mu}{k}\right) v
$$

where $\varphi$ and $\rho$ are the inertial factor and density of the liquid, respectively. This equation changes to Eq.
(5) by substituting $P_{i}\left(\mathrm{~kg} / \mathrm{m}^{4}\right)$ for $\varphi \rho$ and $P_{v}\left(\mathrm{~kg} / \mathrm{m}^{3} \mathrm{~s}\right)$ for $\frac{\mu}{k}$

$$
\frac{\Delta P}{L}=-\left(P_{i} v^{2}+P_{v} v\right)
$$

where $P_{i}$ and $P_{v}$ are the porous inertial and porous viscous resistance coefficients, respectively. These coefficients can be determined using the characteristic curve of the pressure drop vs. fluid velocity obtained experimentally.

Hydraulic filter design requires considering many parameters. Fundamental information can be determined experimentally. Definitive conclusions about the performance of the filter in a real system can be difficult to obtain. Moreover, obtaining information about the flow in the filter, the effect of the filter placement on the flow, and the idle regions where the fluid does not pass can be challenging in experiments. In this study, the oil flow in the hydraulic filter is investigated experimentally and numerically. The flow characteristics in the filter cannot be easily determined via experiments. Therefore, numerical results validated with experimental data are used to examine the flow in the filter. A numerical simulation is an accurate tool for determining the above phenomena. The benefits of simulating the fluid flow in the hydraulic filter can be summarized as follows:

$>$ Flow circulations, which are difficult to detect experimentally, can be easily determined. Thus, the pressure drop is minimized.

$>$ The area over which the fluid passes through in the filter element and flow time can be increased to the maximum by obtaining a uniform flow.

$>$ The contamination time is increased in turn leading to an increased filter life.

$>$ Ideal and efficient hydraulic filter designs can be developed.

In hydraulic filters, the pressure drop is mainly due to the filter element, contaminants, flow, and viscosity of the oil. The novelty of this study is that it reveals the factors that increase the pressure drop leading to inefficient use of the filter. Thus, researchers and filter designers must be aware of for the effects of the flow caused by the structure of the filter. Hence, the flow occurring in hydraulic filter, which is not sufficiently described in existing literature, is examined in the current study.

The remainder of this article is structured as follows. First, the experimental materials and methods are described. Then, the numerical methods, including the geometrical model and boundary conditions, and the mesh structure are defined. Then, the experimental and numerical results are discussed. Finally, the conclusions are presented.

\section{EXPERIMENTAL MATERIALS AND METHODS}

Experimental studies were conducted using the experimental setup shown in Fig. 2. ISO VG 22 
hydraulic oil, whose properties are given in Table 2, was used. The system was operated at flow rates of $0-300 \mathrm{lt} / \mathrm{min}$. The electric motor for the pump was controlled by a driver. Thus, the desired flow rate could be easily adjusted. During the experiments, the flow rate was measured using a Webtec CT300RSR-B-B-6 (with accuracy $\pm 1 \%$ ) flowmeter. A pressure sensor (Webtec SR-PTT-600-05-0C in the range of $0-600$ bar with an accuracy of $0.25 \%$ at full scale) was used to measure the oil pressure and temperature. The data from the sensors were recorded by a Webtec SR-HPM-460-05-0C data logger.

The application of the hydraulic filter in the experiments is shown in Fig. 3. The filter was connected to the vehicle in the opposite direction unlike a standard filter for ease of maintenance when the filter element had to be changed or the upper cover of the filter had to be opened and removed. There is a check valve inside the filter. The check valve in the filter prevents hydraulic oil from spilling outside.

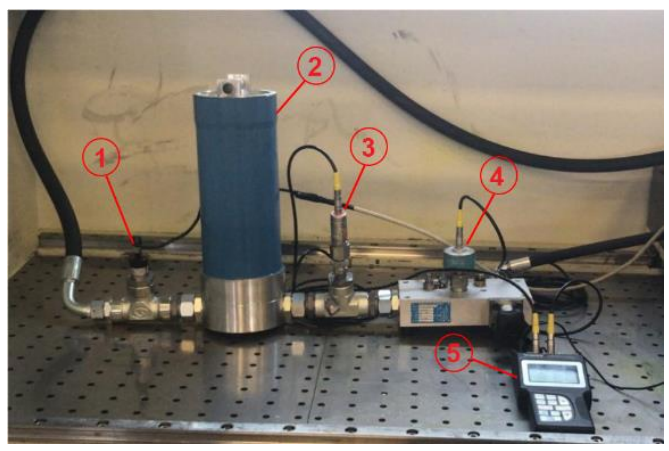

Fig. 2. Experimental setup. 1) output pressure transmitter, 2) hydraulic filter, 3) inlet pressure transmitter 4) flowmeter, and 5) data logger.

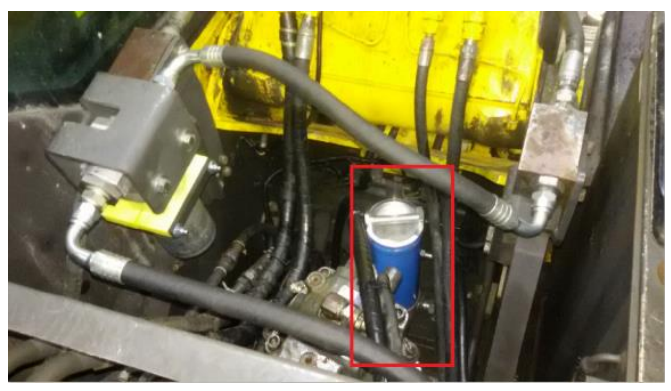

Fig. 3. Application of the used hydraulic filter in an excavator.

Initially, the experiments were performed without the filter element. The tests were repeated with the filter element to determine the porous features of the filter element. Once the temperature of the oil reached $40{ }^{\circ} \mathrm{C}$, which is considered the optimal temperature in hydraulic systems, the experiments were started. This temperature was kept constant throughout the experiments to prevent any changes in the oil viscosity. Experiments were performed at eight different Reynolds numbers $(1117,1257,1397$, 1536, 1676, 1816, 1955, and 2095) for both cases. The porous features of the filter element were determined using the difference between both the pressure drops, as shown in Fig. 4. The velocities presented in this figure were calculated from the flow rate, assuming that the fluid passed through all surfaces of the filter homogeneously. The relationship between the pressure drop and oil velocity was fitted as a curve using the least-squares regression method. The $\mathrm{R}^{2}$ of the regression was 0.97 . The first and second terms of the equation are the porous inertial resistance $\left(P_{i}=9 \times 10^{11} \mathrm{~kg} / \mathrm{m}^{4}\right)$ and porous viscous resistance $\left(P_{v}=1 \times 10^{8} \mathrm{~kg} / \mathrm{m}^{3} \mathrm{~s}\right)$, respectively, as given in Eq. (5). The porosity of the filter element is defined as $3 \%$.

Table 2. Physical properties of the oil.

\begin{tabular}{|l|l|}
\hline Properties & Values \\
\hline Kinematic viscosity & $22 \mathrm{cST}$ \\
\hline Temperature & $40^{\circ} \mathrm{C}$ \\
\hline Density & $860 \mathrm{~kg} / \mathrm{m}^{3}$ \\
\hline
\end{tabular}



Fig. 4. Pressure drop versus velocity of the flow.

\section{NUMERICAL METHODS}

Siemens STAR CCM+ software was used to simulate the flow in the hydraulic filter. The flow was simulated by solving the Navier Stokes equations at steady-state conditions. The SIMPLE algorithm provided the coupling of the pressure and velocity terms, and the second-order upwind scheme was employed to discretize the equations. Laminar flow was assumed because the Reynolds number was less than 2200 for all simulations.

The governing equations of the simulations were the mass and momentum conservation equations, which were solved simultaneously using a coupled flow model as a vector of equations. The velocity field was derived from momentum equations. Pressure was calculated from the continuity equation, and density was evaluated from the state equation. The gas in the hydraulic oil was neglected so that the flow was simulated as a single phase.

The mass conservation equation of incompressible flow is as follows:

$$
\nabla \cdot \vec{V}=0
$$

where $\vec{V}$ is the velocity vector.

The momentum conservation equation is as follows: 


$$
\rho \frac{\partial \vec{V}}{\partial t}+\nabla \cdot(\rho \vec{V} \vec{V})=\overrightarrow{f_{b}}-\nabla p+\nabla \cdot \tau
$$

where $\rho, p$ and $\overrightarrow{f_{b}}$ are the fluid density, static pressure, and resultant body force (e.g. centrifugal and gravitational forces), respectively, and $\tau$ is the viscous stress tensor for Newtonian fluids, as described in Eq. (8):

$$
\tau=\mu\left[\left(\nabla \vec{V}+\nabla \vec{V}^{T}\right)-\frac{2}{3} \nabla \cdot \vec{V} I\right]
$$

where $\mu$ and $I$ are the dynamic viscosity of the liquid and unit tensor, respectively.

\subsection{Geometric Model and Boundary Conditions}

Fig. 5 shows the 3D model of the filter, including the bowl (Fig. 5b), perforated support tube (Fig. 5c), and filter element (Fig. 5d). The 3D CAD model of the hydraulic filter is shown in Fig. 5a is transparent. The space inside the filter was created to obtain the computational domain. Therefore, the perforated support tube was defined as an empty volume, whereas the holes were defined as solids, as shown in Fig. 6. The liquid entering the system was directed to the outlet by passing through these holes. The outer and inner domains were connected to the filter element through the interface. Thus, two regions, including fluid and porous media, were used in the analysis, as shown in Fig. 6. a)

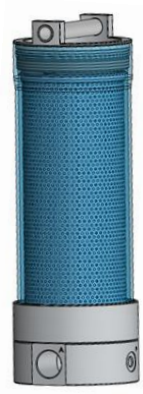

b)

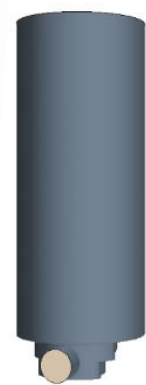

c)

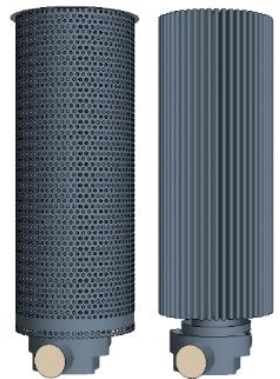

Fig. 5. a) Partially transparent 3D CAD model, b) 3D computational domain, c) perforated support tube, and d) filter element of the hydraulic filter used in the experiments.

Fig. 7 shows the dimensions of the domain. The height and outer diameter of the cylinder domain are 296.4 and $133.0 \mathrm{~mm}$, respectively. The total unfolded length of the outer face of the filter element is $1831.5 \mathrm{~mm}$. Therefore, the surface area of the filter element (i.e., the area where the fluid passes) is 54.3 $\mathrm{cm}^{2}$. The thickness of the perforated support tube is $1 \mathrm{~mm}$. Its outer and inner diameters are 114 and 112 $\mathrm{mm}$, respectively. The outer edge of the filter element is located adjacent to the inner side of the perforated support tube. The thickness of the filter element is $1.5 \mathrm{~mm}$.

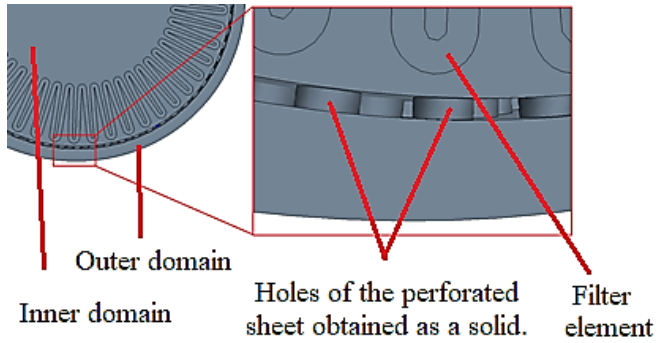

Fig. 6. Computational domain, perforated support tube, and filter element.
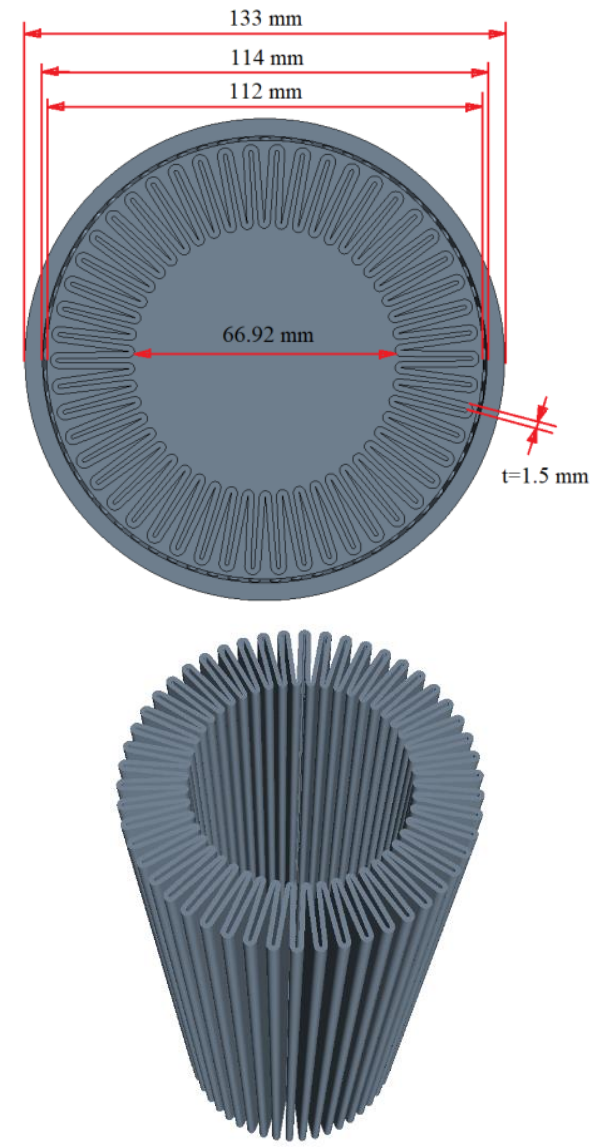

Fig. 7. Dimensions of the 3D domain in the top view.

The mass flow at the inlet and pressure at the outlet was defined to allow the liquid to enter and exit the domain, respectively, as shown in Fig. 8. The diameters of the inlet and outlet boundaries were $30.29 \mathrm{~mm}$. The environmental approach excluded the dynamic head at the boundary, discouraging backflow. No-slip and adiabatic boundary conditions were applied to all other walls.

In this study, a polyhedral mesh was used in the flow simulations through the hydraulic filter, as shown in Fig. 9. The main benefit of a polyhedral mesh is that each cell has many neighbours, and thus gradients can be predicted effectively (Sosnowski et al. 2018). There are a total of 16,042,947 cells, which included $10,131.438$ cells in the fluid domains and 5,911,509 cells in the porous media. There are two conformal 
interfaces at the inner and outer sides of the filter element, as shown in Fig. 9.
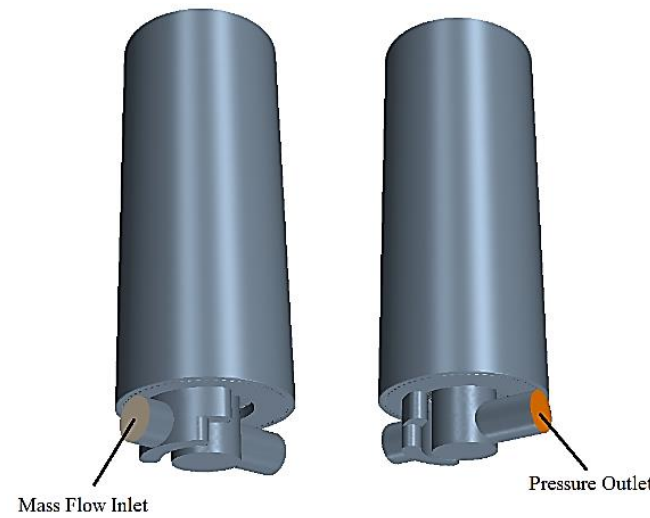

Fig. 8. Boundary conditions of the hydraulic filter.

\subsection{Mesh Domain}


Fig. 9. Polyhedral mesh structure of the hydraulic filter.

Fig. 10 shows the graph of the pressure drop between the inlet and outlet of the hydraulic filter. The pressure drop remained constant after approximately 80 iterations. This event was similar in all other simulations. The simulation was run for approximately 20 additional iterations. Totally 100 iterations were determined sufficient for obtaining the numerical results.



Fig. 10. Change in pressure drop occurring in hydraulic filter with respect to the number of iterations.

\subsection{Validation and Mesh Independence Study}

Fig. 11 shows the pressure drop of the hydraulic filter versus the Reynolds number. The pressure drop between the entrance and exit increases with the Reynolds number, as shown in Fig. 11. The numerical results show close agreement with the experimental data. Thus, these numerical results were used to comprehensively analyze the flow in the filter.

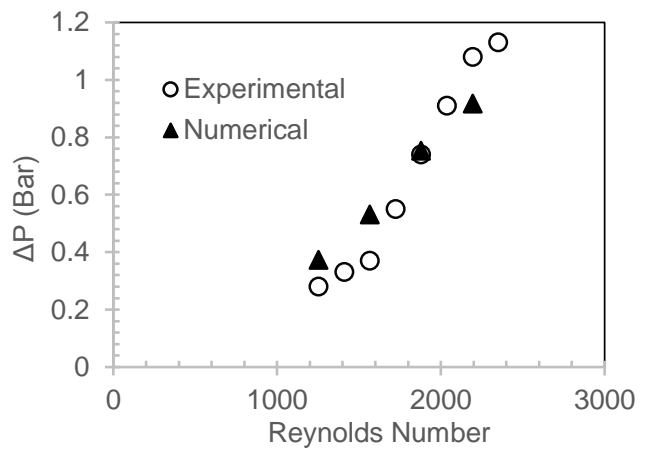

Fig. 11. Pressure drop versus velocity of the flow.

There are two conformed interfaces on both sides of the filter elements. As the filter element had a small thickness/radius and the perforated support had nearly 2022 holes, the conformal interfaces could not be obtained using coarse meshes (total number of cells less than 16,000,000). Therefore, the mesh size, which cannot help in characterizing the flow phenomenon, could not be determined. However, the pressure drop remained nearly unchanged at smaller mesh sizes, as shown in Fig. 12; therefore, this mesh size was considered sufficient to simulate the flow phenomena.

\section{ReSUlts AND Discussions}

In this study, experimental and numerical analyses are performed to examine the flow and pressure drop across the hydraulic filter. As it is extremely difficult to determine the flow phenomena in the hydraulic filter experimentally, the numerical results, which were validated with the experimental data, were used to examine the flow phenomenon. 


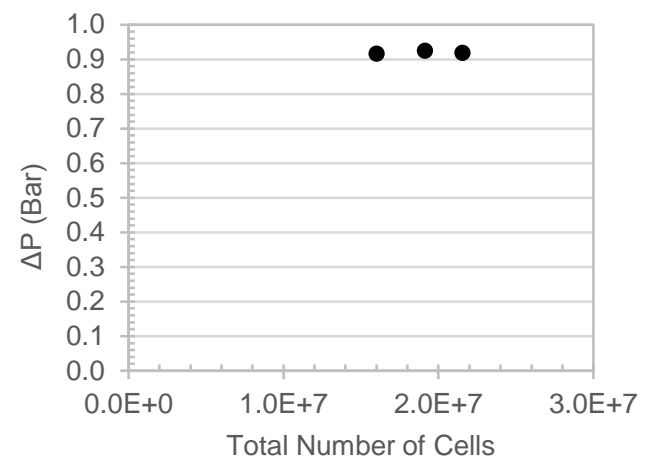

Fig. 12. Pressure loss dependent on the total number of cells for mesh independence study $(\operatorname{Re}=2195)$.
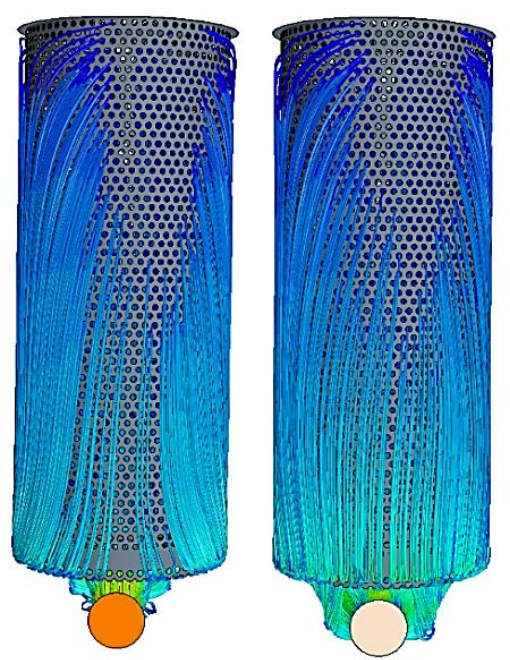

a)



b)

Fig. 13. Velocity magnitude of streamlines around the a) perforated support tube and $b$ ) filter element $(\mathrm{Re}=1955)$.

Fig. 13a and Fig. 13b show the velocity magnitude of the streamlines around the perforated support tube and filter element, respectively. The maximum velocity occurs at the entrance and exit of the hydraulic filter. The flow comes from the outer cylinder of the filter to the filter element through the holes in the perforated support tube. It then passes through the filter element and is directed to the pressure outlet, as shown in Fig. 13. The fluid does not enter the holes on the upper parts of the filter at the entrance and exit sides. Fig. 13 shows that there is no entrance from the lower regions of the entrance side into the filter element. Therefore, the upper regions of the filter element remain idle, and the fluid passes through the filter element mostly in the lower regions.

Figure 14a shows the vertical downward velocities in the mid-plane of the filter, which increase toward the outlet. Downward flow does not occur in the upper parts of the filter. It occurs in the middle of the filter. The velocity of the flow increases toward the exit of the filter. A speed gradient in the regions close to the exit results in Dean vortices (Dean 1927), as shown in Fig. 14b. This is caused by the change in the main movement directions of the liquid particles under centripetal forces. Therefore, two pairs of secondaryflow vortices are formed in the exit region. These secondary flow patterns cause a pressure drop in the filtering system. In addition, the sudden increase in the flow velocity will cause a pressure drop in the corner toward the outlet and may result in cavitation.

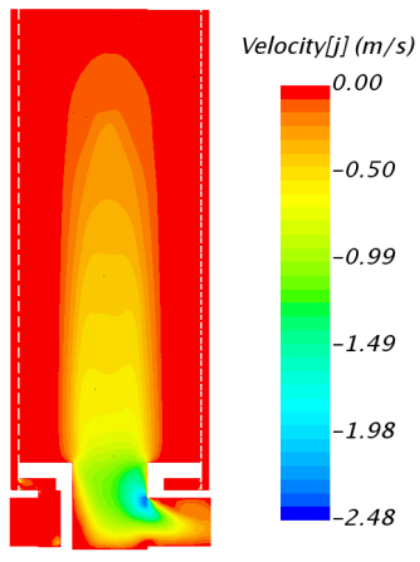

a)

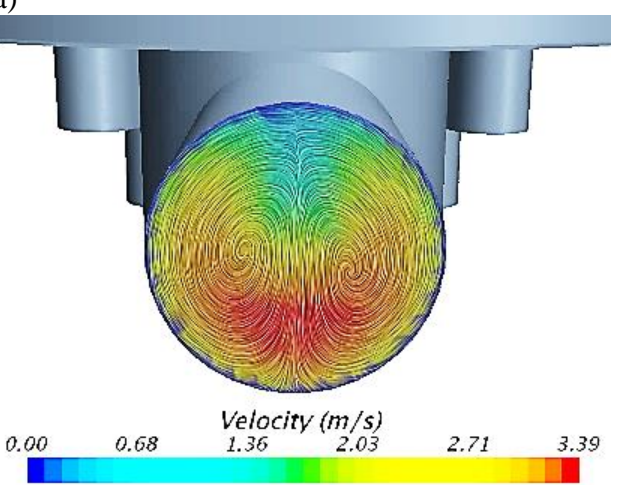

b)

Fig. 14. Velocities of the fluid at the a) mid-plane (vertical downward velocity) and b) outlet of the hydraulic filter. 


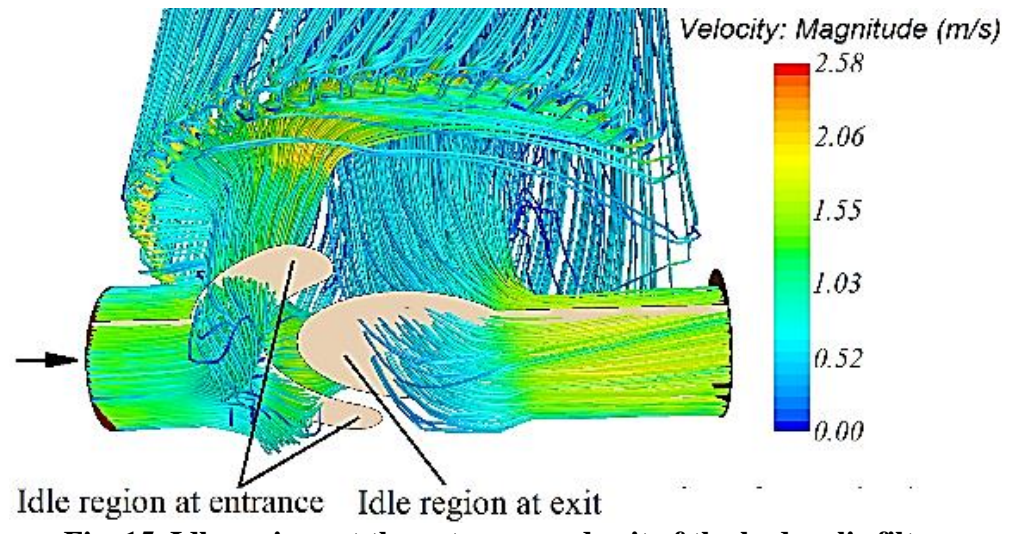

Fig. 15. Idle regions at the entrance and exit of the hydraulic filter.

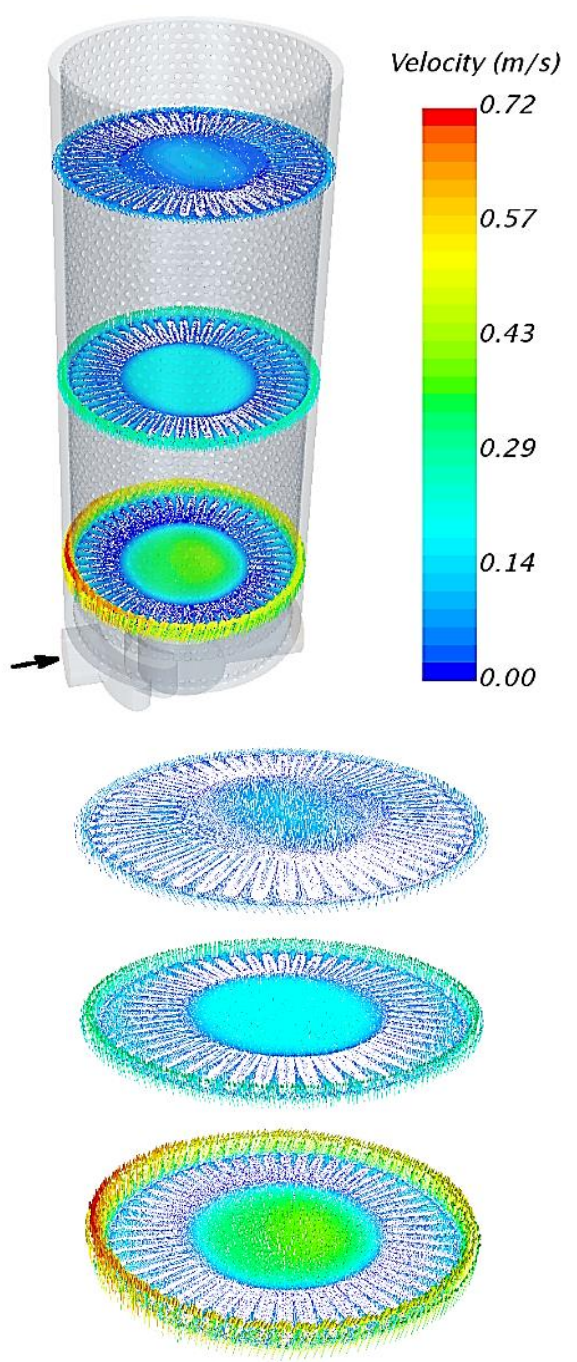

Fig. 16. Velocity vectors in different crosssections.

Idle zones are formed at the entrance and exit of the hydraulic filter, as shown in Fig. 15. The fluid entering the hydraulic filter hits the opposite wall in the filter and divides sideways into two flow directions. It enters the filter without entering the edges of this arc-shaped region. Therefore, these edge regions are unnecessary. Again, the fluid hits the bottom region vertically toward the outlet from the filter, as shown in Fig. 15. Thus, an idle region is formed where vortices occur toward the exit. Sharp changes in the flow direction of the liquid create an unnecessary force on the filter body according to the principle of conservation of momentum and results in energy loss.

The velocity vectors at different sections of the filter are shown in Fig. 16. The fluid reaches the maximum velocity at the inlet radially on the inputoutput side (lower parts). The figure also indicates that the liquid velocity decreases toward the upper parts. The fluid passing through the filter element at the bottom is directed toward the downstream outlet. On the upper side, the fluid passing through the filter element continues flowing radially and is then directed to the downstream outlet at a low speed. This indicates that the flow is concentrated at the lower sides of the hydraulic filter. Thus, the upper parts of the filter remain idle and all areas of the filter element cannot be used homogeneously and effectively.

\section{SUMMARY AND CONCLUSIONS}

In this study, experimental and numerical analyses were conducted to investigate the flow and pressure drop in the hydraulic filter. The flow in the filter was investigated in detail using numerical results validated with the experimental data. The main findings of this study can be summarized as follows:

$>$ The results of the numerical simulations showed that the fluid does not pass through the entire surface of the filter element homogeneously. The entire surface area of the filter element in hydraulic filters should be employed to obtain high performance and durability.

$>$ A considerably low volume of fluid passes through the filter element at the fluid inlet and outlet sides than in the opposite direction. Therefore, this numerical study showed that these regions will remain idle, and contaminants will accumulate in other regions.

$>$ Pressure drop occurs at the outlet of the filter owing to the Dean vortex. This study showed the idle regions of the filter element and 
recirculation regions in hydraulic filters. This work can be continued by other researchers with new designs to effectively use the entire area of the filter element and eliminate recirculation.

$>$ The limitation of this study is that it simulates the situation when a new filter is first operated. Flow phenomena would change as the filter begins to trap and hold the contaminants.

\section{REFERENCES}

Alan, G. and M. Tercan (2013). Hava Filtrasyonu Amaciyla Kullanılan Tekstillerin Verimlilikleri ve Toz Tutma Kapasiteleri. Pamukkale Üniversitesi Mühendislik Bilimleri Dergisi 19(4), 179-186.

Dean, W. R. (1927). XVI. Note on the motion of fluid in a curved pipe. The London, Edinburgh, and Dublin Philosophical Magazine and Journal of Science 4(20), 208-223.

Düz, H. (2019). Numerical and experimental study to predict the entrance length in pipe flows. Journal of Applied Fluid Mechanics 12(1), 155-164.

Eker, O. F., F. Camci and I. K. Jennions (2016). Physics-based prognostic modelling of filter clogging phenomena. Mechanical Systems and Signal Processing 75, 395-412.

Gorle, J. M. R., V. M. Heiskanen, S. Nissi and M. Majas (2018). Effect of temperature, flow rate and contamination on hydraulic filtration. $M M$ Science Journal 2018(October), 2490-2493.

Korkmaz, Y. S., A. Kibar and K. S. Yigit (2021). Experimental and Numerical Investigation of Flow in Hydraulic Elbows. Journal of Applied Fluid Mechanics 14(4), 1136-1146.

Lasseux, D. and F. J. Valdés-Parada (2017). On the developments of Darcy's law to include inertial and slip effects. Comptes Rendus - Mecanique 345(9), 660-669.

Majdan, R., Z. Tkáč, R. Abrahám, K. Kollárová, I. Vitázek and M. Halenár (2017). Filtration systems design for universal oils in agricultural tractors. Tribology in Industry 39(4).

Momin, T., R. P. Chandrasekar, S. Balasubramanian and A. J. Basha (2017). Design and Analysis of High Pressure Hydraulic Filter for Marine Application. IOP Conference Series: Materials Science and Engineering 197(1), 012050.

Okhotnikov, I., K. Abuowda, S. Noroozi and P. Godfrey (2020). Numerical and experimental investigation of the metering characteristic and pressure losses of the rotary tubular spool valve. Flow Measurement and Instrumentation 71, 101679.

Olszak, A., K. Osowski, I. Musiałek, E. Rogoś, A. Kęsy and Z. Kęsy (2020). Application of plant oils as ecologically friendly hydraulic fluids.
Applied Sciences (Switzerland) 10(24), 9086.

Park, M. (2002). Fatigue failure of a hydraulic filter head. Engineering Failure Analysis 9(4), 435450.

Perumal, K. and R. Ganesan (2016). CFD modeling for the estimation of pressure loss coefficients of pipe fittings: An undergraduate project. Computer Applications in Engineering Education.

Reghunathan Valsala, R., S. W. Son, A. Suryan and H. D. Kim (2019). Study on reduction in pressure losses in pipe bends using guide vanes. Journal of Visualization 22(4), 795-807.

Rinaldi, E., J. Canton and P. Schlatter (2019). The vanishing of strong turbulent fronts in bent pipes. Journal of Fluid Mechanics 866, 487502.

Singh, M., G. S. Lathkar and S. K. Basu (2012). Failure Prevention of Hydraulic System Based on Oil Contamination. Journal of The Institution of Engineers (India): Series C, 93(3), 269-274.

Sosnowski, M., J. Krzywanski, K. Grabowska and R. Gnatowska (2018). Polyhedral meshing in numerical analysis of conjugate heat transfer. EPJ Web of Conferences 180, 02096.

Ŝtigler, J., R. Klas, M. Kotek and V. Kopecký (2012). The fluid flow in the t-junction. The comparison of the numerical modeling and piv measurement. Procedia Engineering 39, 19-27.

Sutherland, K. (2008). Filters and Filtration Handbook, Fifth Edition. In Elsevier.

Tarodiya, R., S. Khullar and B. K. Gandhi (2020). CFD modeling of multi-sized particulate slurry flow through pipe bend. Journal of Applied Fluid Mechanics 13(4), 1311-1321.

Tekelioğlu, S., S. Eldek, H. Gümüş, A. Sarıgül, Ş. Ayhan, A. Kahraman and M. Dilmeç (2020). Hidrolik Yüksek Basınç Hattı Filtre Gövdesinin Tasarımı, Optimizasyonu, Üretimi ve Test Edilmesi. Mühendis ve Makina 61(701), 299319.

Wakeman, R. (2007). The influence of particle properties on filtration. Separation and Purification Technology 58(2), 234-241.

Zahedi, P., M. Parsi, A. Asgharpour, B. S. McLaury and S. A. Shirazi (2019). Experimental investigation of sand particle erosion in a $90^{\circ}$ elbow in annular two-phase flows. Wear 438, 203048.

Zhang, R. Cai, Yu, X., Hu, Y. long, Zang, H. Jiao and W. Shu (2018). Active control of hydraulic oil contamination to extend the service life of aviation hydraulic system. International Journal of Advanced Manufacturing Technology 96(5), 1693-1704. 